\title{
Physical Time and Intrinsic Temporality
}

\author{
Georg Franck
}

\begin{abstract}
Physical theories distinguish two notions of time: reversible, homogeneous parameter time (relativity theory and quantum mechanics) and irreversible, directed time (thermodynamics). Both concepts differ fundamentally from what we define implicitly by using the tenses and temporal adverbs in language. The tempora past, present, and future hinge upon one uniquely exposed moment: the now. The now is the moment of actuality in the process of subjective awareness. It proceeds spontaneously and irresistibly in relation to all datable points of time. Without reference to the moving now, past and future only denote directions in time. But there is no physical definition of the now. Physical time, be it reversible or irreversible, differs from subjectively experienced time in that it is atemporal. Because physics has no notion of the now it cannot genuinely treat past and future as temporal regions. As a physicist, Einstein consistently declared the division of time into these regions as illusory. In the first two sections of this paper we reaffirm that Einstein was right on logical grounds. In the third section, however, we insist that the actuality of the now and its movement are truths that logical reason has not the power to question. In the fourth section we shall be looking for a clue to escape the dilemma.
\end{abstract}

\section{Physical Time}

Relativity theory dispelled the notion of absolute simultaneity, which was inherent in the view of an objective, independently definable now. If 
simultaneity is relative to the location of an observer, the now is relative to that location, too. Locations that are spatially distant or distinct with regard to relative motion will differ in time accordingly. If the now is the moment of actuality surrounded by regions of what is no more and not yet actual, locations that differ in time cannot belong to the same actual world. The world as actualized in the now is actual only in the realm of one and the same now. Unsynchronized nows unequivocally belong to different worlds. Thus, temporality splits the universe into as many worlds as there are locations possibly occupied by observers. Einstein's dictum states that this multitude is only subjective and illusory.

Compared with this recent argument there is an age-old one giving rise to a similar conclusion. In the third century B.C., Zeno of Elea set out his riddles of motion which, after a long history of futile efforts to solve them, proved to originate in our notion of the now. Achilles cannot come abreast of the tortoise, the runner in the stadium cannot get started, the arrow must stand still in the air, because for any spatial distance to be traversed there is an infinity of subdistances to be passed. Since each distance, however small, can be subdivided without limit there is an infinity of acts, so the argument, necessary to overcome it. The duration of an infinite number of acts, irrespective of their lengths, always adds to eternity. Thus, motion is impossible.

Zeno's paradoxes deal with a crucial feature of the physical definition of time. Newtonian physics, relativity theory, and standard quantum mechanics all assume that both space and time are continuous as opposed to discrete ${ }^{1}$. By assuming that time is a linear mathematical continuum of instants, physics asserts a time interval to be a dense set of an infinite number of elements. Although it was never seriously questioned that a dense set of spatial elements may add to finite length, it was fiercely debated whether time can be expressed by a dense continuum of numbers ${ }^{2}$. How, this is the question raised by Zeno's

\footnotetext{
1Standard quantum theory has discretized several physical variables whose counterparts in classical physics were mathematically continuous, but space and time remained continuous. In standard quantum theory, every point in continuous space is a potential position of, say, an electron, and every instant of a continuous time is the potential time of a physical event. For a detailed discussion of standard quantum mechanics in its bearing on Zeno's paradoxes see Grünbaum [1], ch. II, § 6 .

${ }^{2} \mathrm{~A}$ mong the most prominent representatives of opposite opinion are Henry Bergson, William James, and Alfred North Whitehead.
} 
paradoxes, can an infinite number of temporal instants be contained in a stretch of finite length?

Perplexing as this question in fact is, it is also astonishing that it was not settled before the mid of our own century. To my knowledge, it was Adolf Grünbaum [1] who first showed that our tacit association of temporal instants with instants being possibly now is the main reason generating the paradox. The now in fact cannot be thought of as an infinitesimally brief instant. The now as the instant of actuality has a positive, non-vanishing, finite duration. An infinitesimally short duration of the now would cause actuality to vanish. Actuality of the now implies non-actuality and actual non-existence of what is not now. For, what is future is not yet existing, what is past is existing no more. The modalities of 'not yet' and 'no more' by no means revoke the negation of existence. If the duration of the now narrows down without limit, actuality dwindles into an extension-less cleft between the actually nonexistent regions of future and past.

For us as conscious beings, the now is of perceivable duration. First, the temporal resolution of sensory awareness is limited. The smallest unit of time perception is about 30 milliseconds. Below $30 \mathrm{~ms}$ perception of the sequence of stimuli, below a somewhat smaller interval (varying with modality) perception of differences as such come to an end. Second, the now as the unit of duration that is experienced as one whole may last up to a few seconds ${ }^{3}$.

Of course, time as a dense set of an infinite number of elements is not constituted by units of this kind. The now itself is not a primitive but a complex concept. It is composed of up to approximately a hundred units of elementary length. Thus, time, as we subjectively perceive it, is quite different from a linear mathematical continuum of instants. Zeno's paradoxes result from not distinguishing these different concepts properly. In this respect, they are proofs of the basic incompatibility of the physical and psychological concepts of time. Grünbaum's resolution of the paradoxes demonstrates that there is a radical and irreducible dissimilarity between time and temporality.

What about the concept of an objective, independently definable now, however? We have already touched upon the problem of such a notion. The

${ }^{3}$ For an overview see Pöppel [2] or Ruhnau [this volume]. 
distinction of past, present, and future as such leaves only what is present as actually existent. Physics usually conceives of the now only as a punctual event or as a point on the time axis. If the present were itself lasting, it would consist of distinguishable earlier and later parts. One and the same now would then include instants just-still-future and just-already-passed. A punctual present, however, has no duration and hence no measurable extension. The reduction of reality to a punctual present leaves us with the void and thus purely abstract picture of a temporally extension-less hypersurface of the universe.

\section{The Logical 'Inexistence' of Temporality}

Grünbaum's resolution of Zeno's paradoxes demonstrates that the physical notion of time in effect has never been about temporality. Physics ever since has adopted an atemporal notion of time ${ }^{4}$. In addition, the concept of temporality was criticized from another point of view. It did not pass without notice that the definition of time given by the usage of tenses and temporal adverbs in language lacks consistency. What is implied in this usage is the definition of the now as something continuously moving relative to all datable, i.e., chronologically fixed, points in time. When we look at the now as being in relative motion there are two options equally odd. First we may consider the now as standing still and the datable points as moving. This movement of all chronology is a strange idea as such. The totality of states of the universe would have to be in ceaseless movement. Not only what is yet future, all the past, too, would have to be in motion. This universal motion is at variance, to say the least, with the changelessness of the past. The second view of relative motion is to consider the now as moving and the datable points as in rest. According to that view, the now propagates like a wave front through the ocean of world states. When the states of the world are in rest, however, nothing is objectively in motion. The moving now, then, is a subjective impression to which nothing except itself is corresponding.

Although this is a somewhat pictorial argument, the dilemma appears with

${ }^{4}$ See, however, section 3 below for a caveat. 
logical strength when we look at the truth value of sentences containing tensed expressions. The sentence "yesterday was Sunday" is true today, Monday, and was false yesterday. The sentence "now it is night" is true tonight and will be false tomorrow morning. The truth value of the predicates "is present", "is past", "is future" changes with time. This change is accomplished by the changing position of the now relative to the once and forever fixed chronology of world states. The now is an indexical expression as is the "here" and are the personal pronouns. The truth value of sentences containing "here", "there", "I", "you" may change in time, too. This change, however, is manageable by modal logic, since the truth value of such sentences does not change if the speaker does not move from the original place of utterance or if nobody else but the original speaker utters the sentence. In contrast, the truth value of sentences with "now" and "then" changes without further ado. It changes spontaneously and irresistibly by the spontaneous and irresistible movement of the now relative to all datable points of time.

Sentences with spontaneously changing truth values are without prospects of scientific approval. On this basis temporality was criticized by Bertrand Russell [3] as early as in 1903. Russell's critique focused on the notion of past, present, and future as properties of moments, states, events, etc. He re-described these properties as concealed relations. An event is past when it happened before the utterance referring to it, it is future when it will happen after this utterance, it is present when it happens simultaneously with the utterance. As far as time goes, the truth of a tensed statement depends on how much earlier or later it is made in relation to its content. In this way, tensed expressions are 'token-reflexive', i.e., bound to the individual specimen of their utterance. Russell suggested to dispense with tenses by replacing them through expressions that relate the moment, event, fact, etc. referred to in explicit terms to the date of the referring utterance. "Now it is night" would then read "At July $5^{\text {th }}, 1993,11$ p.m. it is night", the statement "Yesterday was Sunday" would read "The day before July $5^{\text {th }}, 1993$, is Sunday". Sentences like these have stable truth values. An event that happened before another event will always be earlier; an event that happened after another one will forever be later. Thus, replacement of tenses by relational determinations like 'earlier than', 'simultaneous with', and 'later than' disposes of the problems of unstable truth values.

Russell's remedy is deficient in two respects, however. First, the statement "At 
July $5^{\text {th }}, 1993,11$ p.m. it is night" is equivalent to the statement "Now it is night" if and only if July $5^{\text {th }}, 1993,11$ p.m. is now; the statement "Yesterday was Sunday" is synonymous with the statement "The day before July $5^{\text {th }}, 1993$, is Sunday" if and only if Monday July $5^{\text {th }}, 1993$, is today. Dates must be conventionally fixed to the now, otherwise they are completely arbitrary. Secondly, tenses give expression to the movement of the now whereas relational determinations do not.

Relational determinations remain true or false irrespective of whether time goes by. The changing truth values of tensed statements, on the contrary, show like the hands of a clock that time goes by. Since relational determinations refer to one and the same kind of moments, states, events, etc. as tense does, there is something lacking in the former as compared with the latter.

Instead of reformulating temporality in relational terms, Russell expelled it from language altogether. The perplexities of tense not only survived, but were, in a sense, fostered by this attack. Shortly after Russell's critique, and inspired by its failure, John McT. Ellis McTaggart [4] published a proof claiming to demonstrate the 'unreality of time'5. Arguing that time without temporality is a chimera of abstract thought, McTaggart calls for a blow against the concept of time as such. After asserting that inconsistent concepts cannot denote something real, he contends to show that temporality suffers from incurable inconsistency.

McTaggart's proof starts with a clear exposition of three semantically divergent meanings of time. There is (A) temporality as expressed in terms of past, present, and future, (B) the relational order of moments, states, events, etc. as expressed by the relations 'earlier than', 'simultaneous with', and 'later than', and $(C)$ the mathematical definition of time as a linear continuum of instants. For brevity, the temporal order is denoted as the A series, the relational order as the $B$ series, the continuum of datable points as the $C$ series. The $C$ series is atemporal and only nominally distinct from a further spatial dimension. The B series is atemporal, too, as long as there is no further reference to something that passes. By the relations of 'earlier than', 'simultaneous with', and 'later than' the totality of world states is ordered once and forever ${ }^{6}$. Nothing is changed by the

\footnotetext{
${ }^{5}$ The proof is restituted and amplified by annotations and rejoinders to replies to its first publication in McTaggart [5], ch. 33.

${ }^{6}$ The order, moreover, is unequivocal with regard to one and the same frame of reference.
} 
passage of time. A state that is earlier than another one at any time will stay earlier forever, a state that is at any time later than another one was and will be always later. With the passage of time only the A series changes. What today is future may be present tomorrow and past the day after tomorrow. This change is accomplished by nothing else but the shifting now. It is this shift that essentially distinguishes time from space. This shift, McTaggart contends to proof, cannot be something real.

McTaggart's proof proceeds in two steps. First, he shows that the A series and the $C$ series are fundamental, whereas the B series, being a mixture of both, is derived. Subtraction of any temporal properties from the B series leaves the permanent, space-like order of dated states unchanged. The second step of the proof consists of showing that the A series is contradictory in itself. The reason is that any instant in the A series, when considered from the B series, is as well past as it is present and future. For each of these predicates there are positions in the $B$ series that render them true. What is true once is true forever in the B series. Since the instants contained in the A series are not different from those in the $B$ series, translation from the A series into the B series is neutral with regard to the assignment of temporal properties. An instant being past as well as present and future is, on the other hand, a blatant contradiction.

This apparently simple argument turned out to be of explosive power. For, as easily as the incompatible predicates seem to be made compatible, so notoriously incompatibility recurs. Of course we may translate back into the A series by saying that an instant that is present was future and will be past. But, by saying this, we tacitly introduce a second level of temporalization. To say that an instant was future means that the instants when it is future are past. To say that an instant will be past means that the instants when it is past are future. This reiteration of temporalization amounts to attaching secondary temporal predicates to the primary ones. Inclusion of these secondary predicates now renders nine instead of three predicates. All of these can be predicated to one and the same instant. One and the same instant can be 1 . in the future yet future, 2 . in the future present, 3. in the future past, 4. presently future, 5. presently present, 6 . presently past, 7 . in the past future, 8 . in the past present, and 9. in the past already past. These nine predicates are incompatible again. Resolution of this reentrant contradiction needs the introduction of a third level of instants from which the second level predication is correct. Inclusion of these third level 
properties renders 27 predicates, which are incompatible in turn and so on ad infinitum. Each time we translate back into the A series the contradiction vanishes, each time we re-translate into the B series it recurs.

This is not a sophism but a sophisticated formulation of the bewildering observation that a distinct and unique temporal order, encompassing the whole past and future, belongs to each single instant. With each moment, something present becomes past, and everything having been already past becomes more past. In the same way, each moment something future becomes present, and everything remaining future becomes less future. Each moment the map of all temporality is subject to redesign. McTaggart's proof gives formal expression of the fact that each of these redesigned maps shows different temporal 'colouring' whereas the chronological map of temporary world states remains fixed (at least as long as the same observer is concerned). Both maps, however, do not display different landscapes. The moments that they depict are the same. Yet, the different temporal maps show different properties of one and the same moment. There are maps that show this same moment past, there is (at least) one map that shows it present, and there are other maps that show it future. This multitude of different maps, however, collapses into one and the same chronological map when projected onto the one-dimensional axis of time. This projection is - or at least seems to be - neutral with respect to temporal properties. Nothing that is past or present or future can be made non-past, non-present, non-future, respectively, by mere projection. Thus, the moments keep their presence, pastness, and futurity as elements of the chronological axis. This leads to the contradiction exposed by McTaggart. It can be evaded only by projecting these properties back into the temporal space where they appear in due sequence. By this projection, however, the point could be settled only if the space projected into would be definable in a consistent way. This space is spanned by adding, so to say, a perpendicular temporal axis to the chronological one. The existence of such an axis contradicts the one-dimensionality of time. If resort to higher dimensions is not viable the contradiction arises inevitably. Thus, the nonexistence of higher dimensions of time implies that temporal properties cannot be defined without contradiction. Without temporal properties, however, time is the space-like axis containing the chronological once-and-forever order of states. Time, as we perceive it, becomes logically inconceivable. What is logically inconceivable cannot really exist. Thus, in spite of the strength of its challenge 
and notwithstanding continuing trials of rejection 7 , McTaggart's proof awaits conclusive refutation until now ${ }^{8}$.

It was Kurt Gödel [14] who brought McTaggart's argument into contact with relativity theory. If time consists of the series of nows, only one instantaneous 'time slice' of the universe exists at a moment. Various time slices of this kind, so the moral of McTaggart's proof, cannot co-exist since they bear temporal properties that contradict each other. The very same instant that is yet future in one slice is present in the next and past in the following one. Co-existence of the various slices in effect means that this same moment is as well past as present and future. Nevertheless, in classical terms, the present now and its mapping to past and future are one and the same for various subjects. The now is objective at least in the sense that subjects agree on its intersubjective identity. Even this identity, Gödel reminds, dissolves when simultaneity becomes relative to the location of the observer. The time slices, then, cannot be considered in an intersubjectively unequivocal way. Each observer has his own slice. Since bodily distinct observers are prevented from occupying spatially coinciding locations (at the same time), the time slices of different observers are separated, too.

Difference in spatial location means difference in the instantaneous time slice. Temporality thus, so Gödel argues, lacks objectivity even in the weak sense of intersubjective agreeability. It leaves us with a picture of a universe consisting of as many worlds as there are conscious beings observing this universe from their respective here and now.

\section{The Duality of Time}

Since it is logically true that inconsistent concepts cannot denote something real, it seems logically unavoidable to negate the reality of temporality as expressed by McTaggart's A series. Accordingly, a number of theorists felt obliged to

\footnotetext{
${ }^{7}$ For a summary of various views as to how to reject McTaggart's reasoning see Gale [6], pp. 70-85.

${ }^{8}$ For reconstructions of McTaggart's proof see Broad [7], Dummett [8], Schlesinger [9]-[11], Mellor [12], and Zeilicovici [13].
} 
subscribe the faith in the illusiveness of time as we perceive it. None of them, however, has been practicing this faith. They all were, when measured by their deeds instead of words, satisfied with lip service. Who could ever true-heartedly maintain not to mind whether he has time or not? With having time we mean to dispose of time not yet passed. Or else, who did never ask himself what time it is? The clock time being is the clock time being now. Thus, by his theoretical faith Gödel was not dissuaded form wearing a watch; nor was McTaggart safely prevented from suffering from deadlines. Even Einstein lived as if the past was gone and as if the future was yet to come.

It may be felt that these practical arguments are not quite the point in case yet. Einstein himself underlined the tenacity of the temporal illusion ${ }^{9}$. Moreover, an illusion is something real for its possessor (or the mind possessed by it). An indication for the 'reality' of illusions is how hard it is to get rid of them. Like hallucinations, illusions are apt to present themselves with subjectively overwhelming onset. There are even cases in which it is impossible to tell purely subjective impressions from veridical perceptions. For instance, however strong I may contend that colour is nothing but electromagnetic oscillation, I do not see photons nor wavelengths but qualities: red, blue, green. Knowing that temperature is molecular motion does not prevent me from feeling warmth as that homely quality. Irrespective of the illusiveness of the qualities thus appearing, the generation of the impressions - or 'qualia', as they are denoted - is real as far as the activity providing them is regarded. The making of illusions, like that of qualia, is real in the sense that an inner process resists suppression to imperceptibility. Could it suffice to concede this mind-dependent kind of reality to the moving now in order to save the faith in its objective unreality?

A clear indication of the mind-dependence of the now is the fact that nothing corresponding to it can be measured in the material world. Nowness, in Atmanspacher's parlance ${ }^{10}$, is no exofact. It is something we have notion of by and possibly only by - participation. Participating in nowness, as far as we experience it concretely, is synonymous with being aware. It is not without reason

\footnotetext{
${ }^{9} \mathrm{He}$ even showed seriously worried by the phenomenon of nowness in a conversation reported by Carnap. For reference see the article by Ruhnau in this volume.

${ }^{10}$ See [24] and this volume.
} 
that 'presence' and 'awareness' share a good deal of meaning. There is no nowness when we are dreamlessly asleep or otherwise unconscious, as there are neither redness nor warmth in the sense of qualia. Moreover, I cannot know of the nowness as you experience it. Of course, there are psychological experiments concerning the duration of the now and the impression of its shifting. But these psychological experiments, too, rely on subjective reports of test persons. We are assuming as a matter of course that your nowness feels like mine, as we suppose that your sensations of redness and warmth feel like mine. But we cannot prove the supposed congruence since there is no reliable way of veri- or falsification. We simply have no access to the sensations of the other. We can only agree in denoting the sensation that our neural machinery presents us when processing oscillations in the 600-800 nanometer range as red, as we can agree in denoting the sensation that results from processing molecular pressure above a certain degree as warm ${ }^{11}$.

Obviously, we also agree upon the clock time being now. Relativistic subtleties notwithstanding, this agreement is factual in all practical situations. By being factual it even provides a strong argument for the now being in fact extended. If the now were absolutely instantaneous, i.e., confined to a mathematical point in clock time, bodily distinct subjects never could agree upon its incidence. Each subject, then, would be condemned to inhabit solipsistically one of the worlds of an Everett-like multiverse. By its very existence, sociality suggests that there is, at least in the domain of present awareness, room for the now to be extended. Synchronicity of several nows in the span of subjective awareness does not, however, preclude the hypothetical multitude of worlds as stipulated by the theoretical possibility of narrowing nowness arbitrarily. Since awareness is accessible only from within, the world as it is concretely perceived and imagined is in fact as manifold as there are individual instances of awareness. These worlds are 'window-less' in the sense Leibniz conceived of them in his monadology. Being inaccessible from outside the actuality of awareness renders as many worlds as there are conscious beings occupying their centres consisting

\footnotetext{
${ }^{11}$ By this agreement we can detect, e.g., colour blindness as an anomaly. Positively, this agreement provides us with a means to gauge the semantics of qalia sufficiently well for practical purposes. Wittgenstein's denial of the possibilty of privatelanguages is grounded on the fact that this is the only means of gauging. Putnam's [15] critique of mechanistic theories of meaning is based on the same footing.
} 
in hereness and nowness.

The monads, however, may get limited access to one another if there is a time window sufficiently broad to make the individual nows overlap. Of course, this opening of a time window gives no immediate access to alien consciousness, but it gives way for communication. By simply supposing that my partner is a sentient being like myself, I can interpret her or his expressive behavior as a report about the apparition of her or his world. Of course, this supposition may be illusory again. It is quite conceivable that I am deceived by an automaton that only mimics human behavior (e.g. in a Turing test ${ }^{12}$ ). Yet, there are very good practical - let alone intuitive - reasons to believe that consciousness is operative whenever it makes sense. In these cases we have access to other minds. Further, we are entitled to call nowness something objective in spite of its minddependence. The now is objective, then, in that we can agree intersubjectively upon its incidence and hence upon its actuality without proviso.

Are we approaching a reconciliation of physical time and temporality at this point? There are, to be sure, strong arguments favoring this temporalized version of Leibnizian monadology. On the one hand, it seems to provide a viable way of tackling the relativistic objections to nowness, on the other hand it allows temporality to be perceived as we subjectively perceive it and as we are socially obliged to treat it. Thus, it would be of great comfort if it were sufficient to make the endo- and the exo-view of time compatible. Unfortunately, but not surprisingly, it is not. The resulting problem concerns the interface connecting the endo- and the exoworld. Since any interface shares in both sides, a reconciliation has also to consider the options physics provides in this respect. There remains, to be specific, the quest for an independent explanation of the synchronicity of the individual nows. Second, an account for the intrinsic temporality of the interface is needed.

Why should subjectively different nows, however extended, overlap at all? Why do all aware beings, old and young, use the same time window? Why are we collectively chained to the same now? What is it that selects this moment? Why are we all walking in the same step through the states of the world? What is it that sets the pace? Why are the truth values of tensed sentences changing for

\footnotetext{
${ }^{12}$ It is even conceivable that humans that deny the mechanically irreproducable quality of awareness are automata in this respect. Hans Moravec, are you serious?
} 
us all in the same way? What is it that drives this logical clock? If subjectivity alone was accountable for these accomplishments, physics would have to totally revise its account for subjective powers. It would amount to a miracle if awareness could accomplish this magnificent overall co-ordination by nothing but its own means. How do its individual instances inform each other as to the selection of the moment being now? How do they come to terms concerning its speed of shift? Or is awareness perhaps one whole? ${ }^{13}$

Intersubjective synchronicity is but one aspect of nowness. Another one is its continuous movement. The now is shifting ceaselessly and relentlessly relative to the chronologically once-and-forever fixed positions of time. The time window of subjective awareness does not open without being put in motion at the same time. Only the time slice of the universe that is simultaneous with the present state of the time window can be observed. The ability of perceiving more than one particular slice hinges upon the shift of the window. Without that shift, only some kind of four-dimensional perception could observe larger sectors. Obviously we lack this ability. Even if four-dimensional perception were possible, however, observation of more than the particular sector that consciousness has the capacity to overlook at a time requires relocation of its ramification. Only god-like perception could contemplate the universe as one whole without shifting the window of appearance. If such a shift is occurring, however, it has to be perceived in its own turn for enabling the observer to distinguish between rest and motion on the part of the scene observed ${ }^{14}$. Perception of rest and motion on the part of the observed depends on selfperception of the auto-motion that the observing system is subject to. Conscious observation is inseparably bound to time perception.

Time perception is awareness of the auto-motion of the time window. To this auto-motion no movement in the outer world seems to correspond. Nothing material, no state of the outer world has to change with the relocation of the time window. No change other than that of the moment passing the window is implied with necessity. In this respect it seems quite natural to treat time perception and the perception of qualia alike. Impressions to which no outer

\footnotetext{
${ }^{13}$ As, e.g. Schrödinger [18] speculated.

${ }^{14}$ This corresponds to what Rössler [17] describes as Boscovich covariance of the process of observation and the processes observed.
} 
counterpart corresponds are purely subjective. This equal treatment of time perception and perception of sensual qualities overlooks, however, one striking difference. Time perception is, by its own activity, sharing the property perceived. Whereas the perception of color and warmth does not need to be correspondingly colored or tempered, the perception of temporal change needs temporally changing perceptions. This correspondence of perception and the property perceived is peculiar to time ${ }^{15}$.

Time perception is intrinsically temporal. It actualizes temporal change not only on the part perceived but also on the part of its own history as perceptive activity. Abstraction of temporality from time perception would neglect the object and the occurrence of perception alike. Thus, atemporal concepts of time cannot be based on perception at all. They are products of abstract thought. Of course, a world free of qualia is a product of abstract thought as well. But this abstraction neglects subjective impressions. It restricts itself to the physical counterparts of subjective phenomena. If, however, temporality were abstracted from time perception, no physical counterpart would be left. Subjective awareness is, as far as we know, the only manifestation of the passage of time. Nevertheless, there must be something 'non-subjective' corresponding to it however deep it may be concealed. Assuming that its manifestation has no physical counterpart whatsoever, resort to something 'supernatural' would be unavoidable. A 'meta'-physical principle would be necessary to explain why the access of consciousness to reality is confined to the section of the time window. A mysterious force, not manifesting itself physically, would have to run the logical clock of tense. It would be only in consciousness that we are condemned to live through our bodily states, one after another, and to die some day in the future.

This metaphysical picture fits well into Leibnizian monadology. The monads, as souls, cannot simply cease to exist. They persist in the state of death, as they pre-exist in the state of not yet being brought into their world. They make

\footnotetext{
${ }^{15} \mathrm{Cf}$. Mellor [12], p. 8. Dennett and Kinsbourne [19] object to this view on the basis that there are temporal divergences between the outer application of a stimulus and its conscious perception. The posibility of time-consuming interpretations and rearrangements of stimuli before they become conscious, however, is merely an indication of the projective nature of the conscious now (see sec. 4 below). Time perception is not perception of the sequence of stimuli but awareness of the spontaneous auto-movement of the time window. A voiding reference to the now, Dennett and Kinsbourne are unable to distinguish between perception of succession and time perception.
} 
appearance in life by awaking consciousness. They die, but do not disappear, by its extinction. The world each monad lives in is extended only by its own conscious activity. In the state of death this world has no extension. Dead (or transmigrant) souls are 'metaphysical points' [20]. Being extended by active perception, recollection and expectation, this world consists of the virtual reality of qualia. Appearance and outline of the monadic world is conditioned by the individual capacity of consciousness. Because of its limited capacity, human consciousness can comprehend only one state of the universe at a time. Since there is no physical mechanism ('influxus physicus') as to the selection of the state presented, this selection has to be pre-established by some metaphysical principle harmonizing the presentations to the monads between them. This 'preestablished harmony' is what temporality amounts to if there is no physical selection principle. Since God, the supreme monad, is omniscient, He is not subject to this principle. To Him the universe appears in its totality of states. For God, time is what its physical definition amounts to.

The notion of pre-established harmony is metaphysical in a sense that physics, as an empirical science, cannot acquiesce in. Thus, if the concept of physical time is to be maintained in its standard definition, a second concept of time must be conceded, accounting for what we are experiencing in time perception. We, as humans, have access to time not in the first place by abstract thought, but by being subject to its concrete experience. We may abstract from this primordial experience. Abstracting from what is experienced in time perception, however, means to disregard time perception as such. The intrinsic temporality of time perception prevents separation of activity and result. Thus, in order to arrive at the physical concept of time, a rather strong principle of abstraction must be operative. On the other hand, time perception is a necessary concomitant not only of observation but also of abstract thought. We cannot think conclusively without regard to the auto-moving frame in which the activities of presupposing, drawing inferences, and concluding are performed. Negating temporality as such would amount to negation of the ability of thought, too. To avoid this question-begging consequence, an account of time is needed comprising both what is accessible by abstract thought and what is perceptible by concrete participation. Both notions need to be made compatible in such a way that they do not fall apart dichotomically. Further, it is appealing to relate the way of combination as well as the criterion of distinguishing both 
sides to the endo-/exo-distinction.

Interestingly, in physics there are independent reasons for treating the concept of spacetime in a corresponding manner. The search for non-commuting operators of energy and of time in quantum mechanics has shown that a unitary temporal evolution does not sufficiently reflect the properties requested for an adequate time operator. In this respect it has been suggested by Atmanspacher $[21,23]$ to consider a toy model of (I) a geometry with real space co-ordinates and an imaginary time co-ordinate and (II) another one with imaginary space coordinates and a real time co-ordinate. These geometries are dual in a certain mathematical sense that implies their unity at the same time. Moreover, Atmanspacher brings these dual geometries explicitly into contact with the endo/ exo-distinction. He argues that physics conventionally considers geometry in the spirit of geometry (I). The interpretation he ([24], see also this volume) gives to this fact is that direct empirical access to any concept of time in the sense of its objective operationalization is impossible. Any objectifying observation or measurement of time finally reduces to one or another method of spatial pattern recognition. In this sense, objectification maps imaginary, non-observable, time onto real space. An essential point of Atmanspacher's view of geometry (II) it that time perception is real and hence time perceived. Time perception and time perceived, however, are no exofacts. They satisfy only one of two necessary criteria for operational access: concreteness. They lack externality. It is this mixture of satisfied and unsatisfied criteria that real time shares with sensual qualities.

This interpretation of the dual geometries, implied in the physical notion of space and time, strongly suggests that Einstein's and Grünbaum's verdicts of temporality need modification. It even seems no longer precluded that there is an account of temporality within physics itself. In physics, however, there will be no account of anything that resists consistent definition. The dual geometries furnish only one of several conditions necessary for an account of temporality. Two further ones are that the extension of the present can be conceived in a consistent way and that McTaggart's paradox can conclusively be rejected. We shall address these problems in the following. 


\section{The Geometry of Tense}

The time window of perceptive awareness cannot be narrowed down to the extensionless cleft that the non-presence of past and future seems to claim. Reduction of nowness to exact punctuality would close the window as such. The now must be extended, as it is in fact perceived. If, on the one hand, the now has to be extended, and, on the other hand, nowness cannot contain what is already past and what is yet just to come, time would have to be discrete. This may, as Ruhnau and Pöppel [25] have shown, in a certain sense be the case ${ }^{16}$. But the order of magnitude in this case is different from that of the duration of the now. The now lasts up to three seconds. Time would have to be discretized on this scale. Within this duration the distinction of past, present, and future would have to be precluded. By this preclusion, however, the time window would be compelled to progress leap-wise every three seconds. Since the edges of the time window are blurred such as those of the visual field, it may well be that it progresses step-wise in a pace of about $30 \mathrm{msec}[2,25]$. A leap-wise progression in the order of three seconds, however, contradicts not only introspective but also experimental evidence.

How can the time window be extended without contradiction? Being liable (1) to be extended, (2) to shift quasicontinuously, and (3) to contain no other than unequivocally present sections becomes a paradoxical request if extension is possible in but one dimension. The task, on the other hand, would be patently performable if a second dimension were available. Since it is obvious that there is some recording and recollection of the states exhibited by the time window, the assumption of a dimension used for handling projection should not be considered too exotic. Recollection does not mean time travel back to the event remembered, and anticipation does not mean leaping forward to the date concerned. Both recollection and anticipation are genuine projections. Thus, if for present consciousness there is a screen accessible for projecting what is not present, why then should the just-already-past and the just-yet-future not be projected onto this - virtual - screen, too?

Extension of nowness in this virtual dimension could easily account for

\footnotetext{
${ }^{16}$ For synchronizing sensory input from different modalities there must be certain 'adirectional' temporal zones in the brain.
} 
commonly reported intrasubjective differences regarding the duration of the now. There are numerous reports as to the now becoming dilated in moments of shock or of great pain up to the impression that time comes to a halt. If the time window opens in a virtual dimension it becomes a question of effort or of otherwise mobilized energy how far it widens. It widens by relative retardation of the rear as compared to the propagation of the front edge. Mobilization of additional resources urging the projecting activity may then suffice to slow down the movement of the rear edge up to a momentary halt. By this mobilization the seconds of bursting pain in the dentist's chair or the moment between the 'point of no return' and the crash in an accident may grow to apparent infinity ${ }^{17}$. If the supply of energy to the projective activity decreases with age, a general narrowing of the time window is to be expected as a function of aging. Narrowing the time window may also give rise to the feeling of acceleration of its shift. The feeling that time flows faster with increasing age is commonly reported.

As it seems, there is even experimental evidence for the projective nature of the duration of the now. Libet $[27,28]$ demonstrated in a series of ingenuous experiments that up to $0.5 \mathrm{sec}$ pass between the application of a stimulus to the skin and the beginning sensation of pain. The physiological transmission of the signal to the brain takes only 0.015 seconds. The rest of the time is needed for identifying the sensory input as to what the perception is about. But simultaneously with its emergence the compelling impression arises that the sensation is already lasting for $0.5 \mathrm{sec}$. Consciousness 'ante-dates' the arrival of the sensation. It plays with tricks. These tricks could not be performed if the duration of the now were of other than projective nature.

If time perception relies on this projective ability it should be not too astonishing that it is not operationalizable. It is clear, then, that time as we perceive it is not what its definition as a linear, one-dimensional continuum of datable points is about. Of course, each state of the time screen remains datable. The screen, however, is displaying more instants than the one representing its instantaneous state. It is even conceivable that the date localizing this state in the chronological order of world states is not contained in the section the screen

\footnotetext{
${ }^{17}$ It is even possible to simulate this widening of the time window in machine vision by programs equipped with appropriate dating systems; cf. [29]. By this method the resources needed may be estimated, too.
} 
displays. In spite of ante-dating, there may be a certain lag between the process of presentation and the process presented. There may be even an extrapolative dislocation forward, which might account for our astonishing ability of intuitive precision timing [30]. There may be even an interplay of extrapolative projection and ex-post realignment whose extent depends only on the capacity of the neural machinery furnishing the screen [29]. However this may be in detail, perceived time resembles much more a two-dimensional package of states displaying temporally different events than a one-dimensional continuum of instants.

Each of these states displays instants - of, say, $30 \mathrm{~ms}$ length - that are also displayed by other states. One and the same instant is displayed by different states in a way that attributes slightly different temporal properties to it. The states themselves are different not only in that they have different dates of occurrence in the chronological order, they are also different in that they present the instants they display from a unique perspective. Time perception is awareness of this spontaneously ongoing change of perspective. In order to describe this change consistently, a degree of freedom additional to that allowed by the chronological axis has to be introduced ${ }^{18}$. If the sequential order of the states exhibited by the time screen has to be described as a part of the chronological order of world states, it has to be accounted for as a subset extending in more than one dimension of time.

The secondary dimension, however, is virtual. In a sense, the instants appearing on the screen whose date is not identical with that of the state of the screen displaying them are only a construction of the mind. The borderline between perception and imagination is blurred if not downright suspended in time perception. What is real in time perception is the actual activity focusing the changing perspective. What is apprehended as extending is imaginary - of course, at this stage, not in the mathematical but phenomenological sense. The association, however, does not lead astray in this case. In imagination the extension of the now may be prolonged arbitrarily far beyond the limits set by the actual time screen. This arbitrary prolongation is the first step to the definition of time as a one-dimensional mathematical continuum of instants. The

\footnotetext{
${ }^{18} \mathrm{See}$ [29], [31], [32]. Precursors of the idea are to be found in Dobbs [33] and Salamander [34].
} 
second step consists of abstracting from the now in which this arbitrarily prolonged axis is still centered. This abstraction is indispensable for excluding unstable elements form the definition. Abstraction from the now, however, cuts the connection to time perception. Since the abstraction is indispensable on the one hand, and since it disregards perception on the other hand, the mathematically imaginary time of geometry (I) is imaginary also in the phenomenological sense.

In order to pass to geometry (II) on this phenomenological level this abstraction has to be 'inverted'. Instead of abstracting from the axis containing the instantaneous nows in which the concretely imagined virtual axis is centered, all virtual parts of the time screen have to be disregarded. This abstraction reduces time to the sequence of instantaneous nows that time perception as activity runs through. It may well be that the sequence of these instants is not continuous but discrete ${ }^{19}$. Since no physical account of nowness is available at present [22], we can only speculate about the properties of this axis. One of these speculations is the assumption that the axis of the instants the activity of time perception runs through is orthogonal to the imaginary one. Here again, the phenomenological notions of the real and the imaginary axis would correspond to their mathematical counterparts.

What about temporality with regard to these axes? Its space of possibilities opens when both axes are recombined. First, to come back to the present, it becomes perceivable by the imaginary expansion of the instantaneous now to the width of the time window or, put differently, by combination of the instantaneous now and the projective time screen. The time window, in fact, consists of this combination. Each of its elements bears two co-ordinates: one denoting the position of the state of the window on the real axis and another one denoting the position of the instant displayed in the window on the imaginary axis.

Second, the presentation within the time window is not confined to the present. There are occurring recollections of former states and anticipations of later states as well. By way of these recollections and anticipations we have access to the otherwise inaccessible past and future. According to the duality of their constituents, access to past and future relies on two different abilities. The

\footnotetext{
${ }^{19}$ See Ruhnau/ Pöppel [25] and Ruhnau [26]; further Rössler [16], sec. 7-8.
} 
first, concerning the real axis, is the capacity to store, retrieve, and interpret the states of the time window. Storage means memory, retrieval is the necessary condition of remembrance, interpretation is needed to extract material for the projective construction of anticipated states. The second ability conditioning access to past and future concerns the imaginary axis. It is the capacity of freely choosing the value of the imaginary part of the co-ordinate dating the state actually presented. Free selection of this imaginary component means to be free to leap along the virtual axis in imagination. Only these two abilities in combination provide the necessary and sufficient conditions for living in a world encompassing past and future.

This generative rule of past and future is dual to the logical clockwork of tense. 'Having' past and future presupposes freedom in moving along the virtual axis, whereas a clock that shows the position of the now only makes sense if a degree of freedom concerning movement along the real axis is assumed. Further, remembrance and expectation are re-(or pre-)presentations of past and future only if the date of the record represented or of the event anticipated is supposed to be fixed. Changing truth values of tensed sentences, on the other hand, are indications of a real movement of nowness only if the date of the place at which presentation is possible is supposed to be not arbitrary. Having past and future as regions of a world really lived in indicates that a change of position on the real axis is going on. Changing truth values of semantic expressions, however, indicate that a change of position on the virtual axis is actually taking place. Predication of pastness or futurity to an event means to state that the imaginary component of its dating co-ordinate is smaller or greater, respectively, than its real part (as long as the values increase with time). Stating that the truth values of temporal predicates are changing with time means to discover that the relation between the imaginary and the real part of the dating vector is not fixed.

Thus, Russell was right in showing that temporal properties are concealed relations. But he was not right in believing that the semantics of tense could be stabilized by translation into relational expressions. He overlooked that one of the relata is subject to spontaneous change. Whereas the imaginary component of the dating vector is fixed once and forever, the real part changes with the place in time from which predication is actually possible. Relational determinations are stable only if one fixed, i.e. imaginary, date is combined with another, equally fixed. Only in this case the relational order or, as McTaggart calls it, the B series 
is compatible with the chronological order (or C series). Things change, however, when the relational order comes into contact with the temporal order, i.e. the A series. An event that is later than the now at time $t_{0}$ is simultaneous with it at time $t_{1}$ and earlier than it at time $t_{2}$. Since what is true at one time in the relational order is true forever, this statement comes up to saying that one and the same event is as well future as it is present and past.

Fortunately, this discovery was established by McTaggart as a paradox. Since it still waits for resolution, this paradox provides an excellent opportunity to test the productivity of our enlarged concept of time. Thus, what does it mean to have McTaggart's problem? It means to have to account for temporality without being allowed to make use of an imaginary dimension of time. What McTaggart in fact discovered is that to each instantaneous now a whole temporal order, encompassing all past and future, belongs. He clearly saw that there are as many different temporal orders as there are individual nows. Each of these orders contains the same set of instants. Each order, however, predicates to these same instants different temporal properties. McTaggart tried to account for these differences as differences on the real axis. This account is correct not only, but unavoidable if there is one single axis to map temporal differences onto. Since the spontaneous movement of the now generates the temporal properties of the whole order, change of its position on the real axis implies change of the properties of the elements of this whole axis. If futurity, presence, and pastness are real properties of the elements of the axis, this axis has to change identity each moment. That is what generates the paradox. The elements of the axis cannot change identity without resolving the axis as such. As long as one speaks of an axis its elements have to preserve their identity. If they change identity, then we have in fact something that exists and does not exist at the same time. McTaggart proof is an elaborated version of Heraclitus' dictum "Into the same rivers we step and do not step, we are the same and we are not". What Heraclitus attributes to humans and rivers, McTaggart attributes to time itself. Since time changes whereas the moments, states, events, etc. occurring in it do not, everything occurring in time is in fact future, present, and past. There is no way out in saying that the issue in question was future, is present, and will be past. This translation back into the A series only means to attribute further temporal properties to the same issue. This same issue then is as future yet future, as future present, as future past, as present future, and so on. McTaggart's proof is 
perfectly correct if temporal differences cannot be accounted for as differences conditioned by perspective.

Of course, there is no perspective without at least a second dimension. What McTaggart in fact demonstrates is that it is impossible to account for temporality within a strictly one-dimensional concept of time. Once this is seen the verification is straightforward. To say that something is future or past means to say that it does not exist now. What does not exist now does not really exist. If futurity and pastness are real properties of real moments, states, events, etc., their predication comes up to maintain that the same issue does exist and does not exist. We all know that this contradiction does not prevent us from consistently talking about future and past. The reason is that we all know that something not present can be presently presented in imagination. Future and past exist only in imagination. We have past and future by really imagining what is not really now. Since future and past exist only in present consciousness, they are in fact present and not present at the same time. To be present and not present at the same time is possible only if there is a second, imaginary dimension of time.

This straightforward argument could have easily been accounted for within McTaggart's framework if only someone translated the fundamental A series into the equally fundamental $C$ series instead of the derived $B$ series ${ }^{20}$. To translate a tensed expression into the chronological order of datable points means to date it. Since we know from Russell that expressions predicating temporal properties are token-reflexive, expressions of this kind bear two dates. The first date concerns the moment, event, fact etc. referred to, the second date concerns the speech act that refers. Russell even would have forestalled McTaggart's paradox if he had not treated both dates as fixed. The date of the speech act refers is the date of the 'possibility locus' of predication. This possibility locus is the moving now. Thus, the dating vector of the speech act contains one stable co-ordinate and one that is subject to spontaneous change. Nothing but this instability has to be accounted for to render the translation back and forth between the A series and the $C$ series unproblematic. This mindful translation leaves semantics (as far as time goes) unchanged. Of course, semantics can only account for this instability. The spontaneous change of the

\footnotetext{
${ }^{20}$ For a more detailed presentation of this line of argument see [31].
} 
possibility locus has to be explained independently.

\section{Conclusion}

The concept of time in its standard physical definition requires a strong principle of abstraction. One of the strongest principles of abstraction is the neglect of a whole dimension. The difference between the world that relativity theory describes and the world in which we consciously live is that the former abstracts from the real dimension of time. By this abstraction it arrives at a picture of the world as it is thought to be independent of this same thought. This elegant accomplishment of a seemingly paradoxical task, however, prevents physics from accounting for its own reality as conscious activity and history of thought. In order to account for this reality it does not suffice to switch from geometry (I) to geometry (II). Transgression of a Gödelian borderline is needed. The world behind this borderline has more than one dimension of time.

Acknowledgements

For intensive help concerning both contents and style I am indebted to Harald Atmanspacher. Thanks to Otto E. Rössler for support. This article would not have been written without collaboration with my friend Rachel Salamander long ago.

\section{References}

1. Adolf Grünbaum, Modern Science and Zeno's Paradoxes, London: Allen \& Unwin 1967

2. Ernst Pöppel, Taxonomy of the subjective: An evolutionary perspective, in: J.W. Brown, Neuropsychology of Visual Perception, Hillsdale, N.J.: Erlbaum 1989, pp. 219-32

3. Bertrand Russell, The Principles of Mathematics, Cambridge: Cambridge UP 1903

4. John McT.E. McTaggart, The unreality of time, in: Mind, New Series, no. 68, 1908, pp. 457-74

5. John McT.E. McTaggart, The Nature of Existence, 2 vols., ed. by C.D. Broad, Cambridge: Cambridge UP 1927

6. Richard M. Gale (ed.), The Philosophy of Time, London: Macmillan 1968

7. C.D. Broad, Examination of McTaggart's Philosophy, Cambridge 1938; 
reprint New York: Octagon 1976

8. Michael Dummett, A defence of McTaggart's proof of the unreality of time, in: Philosophical Review, vol. 69, 1960, pp. 497-504

9. George N. Schlesinger, The structure of McTaggart's argument, in: Review of Metaphysics, vol. 24, no. 4, 1971, pp. 668-77

10. George N. Schlesinger, Aspects of Time, Indianapolis: Hackett 1980

11. George N. Schlesinger, How time flies, in: Mind, vol. 91, 1982, pp. 501-23

12. David H. Mellor, Real Time, Cambridge: Cambridge UP 1981

13. David Zeilicovici, Eine (Auf)lösung des Paradoxes von McTaggart, in: Ratio, vol. 28, no. 2, 1986, pp. 159-178

14. Kurt Gödel, A remark about the relationship between relativistic theory and idealistic philosophy, in: Albert Einstein, Philosopher-Scientist, ed. by Paul A. Schilpp, Open Court; LaSalle 1949, pp. 555-62

15. Hilary Putnam, The meaning of "meaning", in: Keith Gunderson (ed.), Language, Mind, and Knowledge, Minnesota: Univ. of Minnesota Press 1975, pp. 131-93

16. Otto E. Rössler, Into the same rivers we step and do not step, we are the same and we are not - On the origin of the now, in: Tagungsband "Chaos und Pädagogik", ed. by J.R. Bloch, Kiel 1993; reprinted in German in: Otto E. Rössler, Endophysik, Berlin: Merve 1992

17. Otto E. Rössler, Boscovich covariance, in: J.L. Casti and A. Karlqvist (eds.), Beyond Belief: Randomness, Prediction and Explanation in Science, Boca Raton: CRC Press 1991; reprinted in German in: Otto E. Rössler, Endophysik, Berlin: Merve 1992

18. Erwin Schrödinger, Das arithmetische Paradoxon - Die Einheit des Bewußtseins, in: Geist und Materie, Braunschweig 1959

19. Daniel C. Dennett/ Marcel Kinsbourne, Time and the observer: The where and when of consciousness in the mind, in: Behavioral and Brain Sciences, vol. 15, 1992, pp. 183-247

20. Gottfried Wilhelm Leibniz, Neues System der Natur und der Verbindung der Substanzen sowie der Vereinigung zwischen Seele und Körper, 1695, in: Fünf Schriften zur Logik und Metaphysik, ed. by Herbert Herring, Stuttgart: Reclam 1966

21. Harald Atmanspacher/ Herbert Scheingraber, A fundamental link between system theory and statistical mechanics, in: Foundations of Physics, vol. 17, no. 9,1987

22. Harald Atmanspacher, The aspect of information production in the process of observation, in: Foundations of Physics, vol. 19, 1989, pp. 553-77

23. Harald Atmanspacher, Complementarity of structure and dynamics, in: $\mathrm{H}$. Atmanspacher / H. Scheingraber, Information Dynamics, New York: Plenum Press 1991

24. Harald Atmanspacher, Die Vernunft der Metis, Stuttgart: Metzler 1993

25. Eva Ruhnau/ Ernst Pöppel, Adirectional temporal zones in quantum physics and brain physiology, in: International Journal of Theoretical Physics, vol. 30, no. 8, 1991, pp. 1083-89

26. Eva Ruhnau, Zeit - das verborgene Fenster der Kognition, in: Kognitionswissenschaften, vol. 2, 1992, pp. 171-79

27. Benjamin Libet, Electrical stimulation of cortex in human subjects, and conscious memory aspects, in: Handbook of Sensory Physiology, vol. 2, ed. by A. Iggo, Heidelberg: Springer 1973

28. Benjamin Libet, The experimental evidence for subjective referral of a sensory experience backwards in time. Reply to P.S. Churchland, in: Philosophy of Science, vol. 48, 1981, pp. 182-97 
29. Georg Franck, Virtual time - Can subjective time be programmed?, in: Ars Electronica 1990, ed. by Gottfried Hattinger, Morgan Russel, Christine Schöpf, and Peter Weibel, Linz 1990, vol. 2, pp. 57-81

30. Dorothea Franck/ Georg Franck, Zwischenmenschliche Verhandlung versus intersubjektive Norm. Für eine Analytik des Sprecherwechsels als nicht regelgeleiteter Selbstorganisation des Gesprächs, in: Papiere zur Linguistik, no. 35/2, 1986, pp. 55-78

31. Georg Franck, Das Paradox der Zeit und die Dimensionszahl der Temporalität, in: Zeitschrift für Philosophische Forschung, vol. 43, no. 3, 1989, pp. 449-71

32. Georg Franck, Die zeitliche Differenz von Natur und Geist, in: Merkur no. 500, 1990. pp. 927-38

33. H.A.C. Dobbs, The dimensions of the sensible present, in: J.T. Fraser/ F.C. Haber/ G.H. Müller (eds.), The Study of Time, Berlin: Springer 1972, pp. 27492

34. Rachel Salamander, Zeitliche Mehrdimensionalität als Grundbedingung des Sinnverstehens, Bern: Peter Lang 1982 